\title{
On a Semi-Linear Wave Equation Associated with Memory Conditions at the Boundaries: Unique, Existence and Regularity
}

\author{
Út V. Lê
}

Communicated by S.-T. Yau, received August 18, 2008.

\begin{abstract}
In this paper the unique existence and regularity of the weak solution of an initial-boundary value problem relating to a semi-linear wave equation and two integral equations at the boundaries are given.
\end{abstract}

\section{Contents}

1. Introduction

2. Preliminary results and notations 316

3. Unique solvability 317

4. The regularity of the weak solution $\quad 322$

References

\section{Introduction}

We study the solution $u(x, t)$ of following semi-linear equation

$$
\begin{aligned}
\frac{\partial^{2} u}{\partial t^{2}}(x, t)-\frac{\partial}{\partial x}\left(\mu(x, t) \frac{\partial u}{\partial x}(x, t)\right) & +G(u(x, t)) \\
& +H\left(\frac{\partial u}{\partial t}(x, t)\right)=F(x, t)
\end{aligned}
$$

1991 Mathematics Subject Classification. 35L15, 35L20; 35L70.

Key words and phrases. Contracted procedure, semi-linear wave equation, unique existence and regularity.

The author is currently supported by the Academy of Finland and Emil Aaltonen Foundation.

(C)2008 International Press 
where $0<x<1,0<t<T$, associated with initial-boundary values given by

$$
\begin{gathered}
\mu(0, t) \frac{\partial u}{\partial x}(0, t)=g_{0}(t)+\int_{0}^{t} k_{0}(t-s) u(0, s) d s \\
-\mu(1, t) \frac{\partial u}{\partial x}(1, t)=g_{1}(t)+\int_{0}^{t} k_{1}(t-s) u(1, s) d s, \\
u(x, 0)=u_{0}(x), \quad \frac{\partial u}{\partial t}(x, 0)=u_{1}(x),
\end{gathered}
$$

where $\mu, G, H, F, g_{0}, k_{0}, g_{1}, k_{1}, u_{0}$ and $u_{1}$ are given real functions satisfying conditions specified later. The terms $G(u(x, t)), H\left(\frac{\partial u}{\partial t}(x, t)\right)$ are, respectively, called the damping, the source term of Eq. (1.1), and we shortly call the sum $G(u(x, t))+H\left(\frac{\partial u}{\partial t}(x, t)\right)$ the damping-source term.

The problems of wave equations associated with memory conditions or integral equations at the boundaries have interested many mathematicians (see [5], [10]$[20],[22],[24],[25])$.

When $\mu(x, t) \equiv 1$ and $F(x, t)=0$, in [10], Nguyen and Alain considered problem $(1.1),(1.4)$ in the case of the full nonlinear damping-source term of $u$ and $\frac{\partial u}{\partial t}$ associated with the homogeneous boundary at $x=0$ and the non-homogeneous boundary condition at $x=1$ given by

$$
-\frac{\partial u}{\partial x}(1, t) \equiv Q(t)=h u(1, t)-g(t)-\int_{0}^{t} k(t-s) u(1, s) d s,
$$

where $h$ is a positive constant; $Q, g$ and $k$ are given functions. We note that (1.5) is deduced from a Cauchy problem for an ordinary differential equation at the boundary $x=1$ as follows

$$
\left\{\begin{array}{l}
Q^{\prime \prime}(t)+\omega^{2} Q(t)=h \frac{\partial^{2} u}{\partial t^{2}}(1, t), t \in(0, T), \\
Q(0)=Q_{0}, Q^{\prime}(0)=Q_{1}
\end{array}\right.
$$

where $\omega>0, Q_{0}$ and $Q_{1}$ are given constants. This problem is a mathematical model describing the shock of a rigid body and a nonlinear viscoelastic bar resting on a rigid base. In this article, the authors obtained the unique solvability of the weak solution.

In [20], Santos studied the asymptotic behavior of the solution of problem (1.1), (1.2), (1.4) in the case of $\mu(x, t) \equiv \mu(t), G(u)=H\left(\frac{\partial u}{\partial t}\right)=0, F(x, t)=0$ associated with a boundary condition of memory type at $x=1$ as follows

$$
u(1, t)+\int_{0}^{t} g(t-s) \mu(s) \frac{\partial u}{\partial x}(1, s) d s=0, t>0,
$$

in which $g$ and $\mu$ are given functions. It is noted that the boundary conditions (1.5) and (1.7) are similar since their formal differences can be crossed out after solving the Volterra equation with respect to the variable $u(1, t)$ given by $(1.7)$.

In $[\mathbf{1 6}, \mathbf{1 7}, \mathbf{1 8}]$, Nguyen, Lê and T. Nguyen considered the unique existence, stability, regularity in time variable and asymptotic expansion for the solution of problem (1.1)-(1.4) when $\mu(x, t) \equiv \mu(t), G(u)=K u, H\left(\frac{\partial u}{\partial t}\right)=\lambda \frac{\partial u}{\partial t}$ and the boundary condition (1.2) is homogeneous and the boundary value at $x=1,(1.3)$, is

$$
-\mu(t) \frac{\partial u}{\partial x}(1, t)=g(t)+K_{1}(t) u(1, t)+\lambda_{1}(t) \frac{\partial u}{\partial t}(1, t)+\int_{0}^{t} k(t-s) u(1, s) d s
$$


where $g, K_{1}, \lambda_{1}$ and $k$ are given functions.

In the above articles, the authors mainly applied Faedo-Galerkin approximation to study the unique solvability.

In the case of homogeneous boundaries, in [21], Sengul investigated the existence of the global attractor of Eq.(1.1) in the case of

$$
\mu(x, t)=1, H\left(\frac{\partial u}{\partial t}\right)=\alpha \frac{\partial u}{\partial t}, \alpha>0
$$

associated homogeneous boundary conditions and the initial conditions similar to (1.4). In [1], Aassila and Benaissa obtained the global unique solvability, also by Faedo-Galerkin approximation, and the decay for the solution of the following problem:

$$
\left\{\begin{array}{l}
\frac{\partial^{2} u}{\partial t^{2}}-\Phi\left(\int_{\Omega} \sum_{i=1}^{n}\left(\frac{\partial u}{\partial x_{i}}\right)^{2} d x\right) \sum_{i=1}^{n} \frac{\partial^{2} u}{\partial x_{i}^{2}} \\
\quad+g\left(\frac{\partial^{2} u}{\partial t^{2}}\right)+f(u)=0 \text { in } \Omega \times[0,+\infty[, \\
u(x, t)=0 \text { on } \Gamma \times[0,+\infty[, \\
u(x, 0)=u_{0}(x), \frac{\partial u}{\partial t}(x, 0)=u_{1}(x) \text { on } \Omega,
\end{array}\right.
$$

for $\Omega$ a bounded domain in $\mathbb{R}^{n}$ with a smooth boundary $\partial \Omega=\Gamma$, where $\Phi, g, f$, $u_{0}$ and $u_{1}$ are given functions. In [4], Benaddi and Rao obtained the energy decay rate of the solution by a shooting method for problem (1.1)-(1.4) where $\mu(x, t)=1$, $G$ and $H$ are linear, $F(x, t)=0$, and $g_{0}=g_{1}=k_{0}=k_{1} \equiv 0$ (or (1.2)-(1.3) are homogeneous). In addition, Phung [19] studied the stabilization of the wave equation with a localized linear dissipation in a three-dimensional bounded domain on which exists a trapped ray given by

$$
\left\{\begin{array}{l}
\frac{\partial^{2} u}{\partial t^{2}}-\sum_{i=1}^{3} \frac{\partial^{2} u}{\partial x_{i}^{2}}+\alpha(x) \frac{\partial u}{\partial t}=0 \text { in } \Omega \times \mathbb{R}^{+}, \\
u(x, t)=0 \text { on } \partial \Omega \times \mathbb{R}^{+}, \\
u(x, 0)=u_{0}, \frac{\partial u}{\partial t}(x, 0)=u_{1}(x) \text { in } \Omega,
\end{array}\right.
$$

for $\Omega$ a bounded domain in $\mathbb{R}^{3}$ with a boundary $\partial \Omega$ at least Lipschitz; $\alpha, u_{0}$ and $u_{1}$ are given functions.

Regarding Mikusiński calculus, D. Takači and A. Takači studied the existence of the solution of problem (1.1), (1.4) in the field of Mikusiński when $\mu(x, t)=1$, $G$ and $H$ are linear, and the boundary conditions are non-homogeneous.

In this paper, we apply a contracted procedure (see $[\mathbf{8}],[\mathbf{1 3}],[\mathbf{2 3}]$ ) to obtain the unique solvability of problem (1.1)-(1.4), and it is believed that the essential proofs must be shorter and easier than what has been brought up since the difficulties relating to the nonlinear damping source can be solved by a suitable contraction. What we obtain here is considered as both the generalization and a more effective approach of those in Aassila and Benaissa [1], in Benaddi and Rao [4], Long and Dinh [10], in Phung [19], in Santos [20], in Sengul [21], D. Takači and A. Takači $[\mathbf{2 2}]$ and in mine [16]-[18], [24], [25].

Furthermore, we can affirm that this work exceeds a routine application of usual methods, namely the Faedo-Galerkin method with the compactness argument and the monotone operator method, for semi-linear damped wave equations as 
popularized by Jacques-Louis Lions several years ago (see [9], [15]) and also by Songmu Zheng [27].

\section{Preliminary results and notations}

First we introduce some preliminary results and notations used in this paper. We omit the definitions of usual function spaces: $C^{m}, L^{p}, W^{m, p}, H^{m}$ for $p \in[1,+\infty]$ and $m \in \mathbb{N}$.

We denote by $\langle\cdot, \cdot\rangle$ the scalar product in $L^{2}(0,1)$ or pair of dual scalar product of a continuous linear functional with an element of a function space. We denote by $\|\cdot\|_{X}$ the norm of a Banach space $X$ and by $X^{\prime}$ the dual space of $X$. We denote by $L^{p}(0, T ; X), 1 \leq p \leq \infty, T>0$, the Banach space of the real measurable functions $v:(0, T) \rightarrow X$, such that

$$
\|v\|_{L^{p}(0, T ; X)}=\left(\int_{0}^{T}\|v(t)\|_{X}^{p} d t\right)^{1 / p}<\infty \text { for } 1 \leq p<\infty,
$$

and

$$
\|v\|_{L^{\infty}(0, T ; X)}=\underset{0<t<T}{\operatorname{esssup}}\|v(t)\|_{X} \text { for } p=\infty .
$$

In addition, we denote by $W^{m, p}(0, T ; X)$ for $p \in[1,+\infty]$ and $m \in \mathbb{N}$ the Sobolev space of all functions $v \in L^{p}(0, T ; X)$ such that $v^{(m)}$ exists in the weak sense and belongs to $L^{p}(0, T ; X)$ and

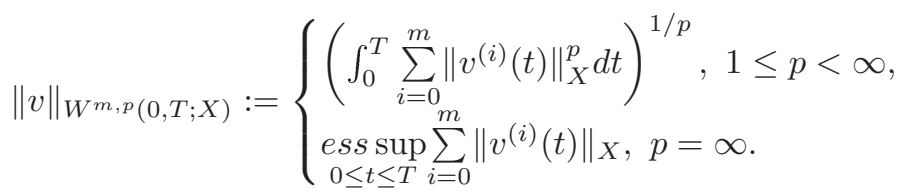

Shortly we write

$$
H^{m}(0, T ; X)=W^{m, 2}(0, T ; X) .
$$

Furthermore, we denote by $C([0, T] ; X)$ all of continuous functions

$$
v:(0, T) \rightarrow X
$$

with

$$
\|v\|_{C([0, T] ; X)}:=\max _{0 \leq t \leq T}\|v(t)\|_{X}<\infty
$$

and $C^{1}([0, T] ; X)$ all of differential functions

$$
v:(0, T) \rightarrow X
$$

with

$$
\|v\|_{C^{1}([0, T] ; X)}:=\max _{0 \leq t \leq T}\left(\|v(t)\|_{X}+\left\|\frac{\partial v}{\partial t}(t)\right\|_{X}\right)<\infty .
$$

In $H^{1}(0,1)$, we use the norm

$$
\|v\|_{H^{1}(0,1)}=\sqrt{\|v\|_{L^{2}(0,1)}^{2}+\left\|v^{\prime}\right\|_{L^{2}(0,1)}^{2}}, \quad v \in H^{1}(0,1) .
$$

Then we have the following lemma whose proof is omitted:

LEMMA 1. The embedding $H^{1}(0,1) \hookrightarrow C^{0}([0,1])$ is compact and

$$
\|v\|_{C^{0}([0,1])} \leq \sqrt{2}\|v\|_{H^{1}(0,1)}
$$

for all $v \in H^{1}(0,1)$. 
Moreover, let a real function $u=u(x, t)$ for $(x, t) \in(0,1) \times(0, T)$ such that $u(t) \in X$ where $X$ is a real Banach space defined on $(0,1)$, there are two following results whose proofs are able to be seen in $[\mathbf{8}]$ :

Lemma 2. Let $u \in W^{1, p}(0, T ; X)$ for some $1 \leq p \leq \infty$. Then

$$
u \in C([0, T] ; X) .
$$

Lemma 3. Suppose $u \in L^{2}\left(0, T ; H^{2}(0,1)\right)$, with $\frac{\partial u}{\partial t} \in L^{2}\left(0, T ; L^{2}(0,1)\right)$. We have

$$
u \in C\left([0, T] ; H^{1}(0,1)\right) .
$$

\section{Unique solvability}

First and foremost, we make some following essential assumptions:

$\left(A_{\mu}\right) \frac{\partial \mu}{\partial t} \in L^{1}\left(0, T ; L^{\infty}(0,1)\right), \mu(x, t) \geq \mu_{0}>0 ;$

$\left(A_{G H}^{(1)}\right) G, H \in L^{2}(\mathbb{R})$;

$\left(A_{G H}^{(2)}\right) \exists K_{G}, K_{H}>0$ :

$$
\begin{aligned}
|G(u)-G(v)| & \leq K_{G}|u-v|, \\
|H(u)-H(v)| & \leq K_{H}|u-v|,
\end{aligned}
$$

for $u, v \in \mathbb{R}$

$\left(A_{F}\right) F \in L^{2}((0,1) \times(0, T))$;

$\left(A_{g}\right) g_{0}, g_{1} \in H^{1}(0, T)$

$\left(A_{k}\right) k_{0}, k_{1} \in W^{1,1}(0, T)$;

$\left(A_{u}\right) u_{0} \in H^{1}(0,1), u_{1} \in L^{2}(0,1)$.

In this paper, we say that a function

$$
u \in H^{1}\left(0, T ; L^{2}(0,1)\right) \cap L^{\infty}\left(0, T ; H^{1}(0,1)\right)
$$

is a weak solution of problem (1.1)-(1.4) iff

$$
\left\{\begin{aligned}
& \frac{d}{d t}\left\langle\frac{\partial u}{\partial t}(t), v\right\rangle+\left\langle\mu(t) \frac{\partial u}{\partial x}(t), v^{\prime}\right\rangle+Q_{0}(t) v(0)+Q_{1}(t) v(1) \\
&+\left\langle G(u(t))+H\left(\frac{\partial u}{\partial t}(t)\right), v\right\rangle=\langle F(t), v\rangle \\
& u(x, 0)=u_{0}(x), \frac{\partial u}{\partial t}(x, 0)=u_{1}(x) \\
& Q_{0}(t)=g_{0}(t)+\int_{0}^{t} k_{0}(t-s) u(0, s) d s \\
& Q_{1}(t)=g_{1}(t)+\int_{0}^{t} k_{1}(t-s) u(1, s) d s
\end{aligned}\right.
$$

for each $v \in H^{1}(0,1)$ and a.e. time $0 \leq t \leq T$, where $\mu(t) \equiv \mu(\cdot, t)$ and $F(t) \equiv$ $F(\cdot, t)$. In this case we can say that problem (1.1)-(1.4) is weakly solvable in $H^{1}\left(0, T ; L^{2}(0,1)\right) \cap L^{\infty}\left(0, T ; H^{1}(0,1)\right)$.

Then, we have the following theorem:

Theorem 1. Let $\left(A_{\mu}\right),\left(A_{G H}^{(1)}\right),\left(A_{G H}^{(2)}\right),\left(A_{F}\right),\left(A_{g}\right),\left(A_{k}\right)$ and $\left(A_{u}\right)$ hold. Then, for $T>0$, the problem (1.1)-(1.4) has a unique weak solution $u(x, t)$ satisfying

$$
u \in H^{1}\left(0, T ; L^{2}(0,1)\right) \cap L^{\infty}\left(0, T ; H^{1}(0,1)\right) .
$$

Proof. The proof consists of two steps as follows.

Step 1. The solvability in $H^{1}\left(0, T ; L^{2}(0,1)\right)$.

Let an operator $\uplus$ be defined as follows. For a given function

$$
u \in H^{1}\left(0, T ; L^{2}(0,1)\right),
$$


set

$$
£(x, t):=G(u(x, t))+H\left(\frac{\partial u}{\partial t}(x, t)\right)-F(x, t) \text { for }(x, t) \in[0,1] \times[0, T] .
$$

From $\left(A_{G H}^{(1)}\right)$ and $\left(A_{F}\right)$, we deduce that

$$
£ \in L^{2}((0, T) \times(0,1)) .
$$

We have the following lemma.

LEMMA 4. With the presence of (3.3) and assumptions $\left(A_{\mu}\right),\left(A_{g}\right),\left(A_{k}\right),\left(A_{u}\right)$, the linear initial-boundary value problem given by

$$
\left\{\begin{array}{l}
\frac{\partial^{2} w}{\partial t^{2}}-\frac{\partial}{\partial x}\left(\mu(x, t) \frac{\partial w}{\partial x}\right)+£=0 \text { in }(0,1) \times(0, T), \\
\mu(0, t) \frac{\partial w}{\partial x}(0, t)=Q_{0 w}(t), \\
-\mu(1, t) \frac{\partial w}{\partial x}(1, t)=Q_{1 w}(t), \\
w(x, 0)=u_{0}(x), \frac{\partial w}{\partial t}(x, 0)=u_{1}(x) \\
Q_{0 w}(t)=g_{0}(t)+\int_{0}^{t} k_{0}(t-s) w(0, s) d s \\
Q_{1 w}(t)=g_{1}(t)+\int_{0}^{t} k_{1}(t-s) w(1, s) d s
\end{array}\right.
$$

has a unique weak solution $w(x, t)$ such that

$$
w \in H^{1}\left(0, T ; L^{2}(0,1)\right) \cap L^{\infty}\left(0, T ; H^{1}(0,1)\right) .
$$

In addition, $w(x, t)$ satisfies the estimate

$$
\left\|\frac{\partial w}{\partial t}(t)\right\|_{L^{2}(0,1)}^{2}+\left\|\sqrt{\mu(t)} \frac{\partial w}{\partial x}(t)\right\|_{L^{2}(0,1)}^{2} \leq \bigodot_{0} \text { for all } t \in[0, T]
$$

for which (C) $_{0}$ is a positive constant independent of $t$.

Since (3.4) is a linear problem, the proof of this lemma is really simple and is similar to what in [14].

REMARK 1. The unique solvability of problem (3.4) is independent of assumption $\left(A_{G H}^{(2)}\right)$.

It is clear that $w$ satisfies the variational problem

$$
\left\{\begin{array}{l}
\frac{d}{d t}\left\langle\frac{\partial w}{\partial t}(t), v\right\rangle+\left\langle\mu(t) \frac{\partial w}{\partial x}(t), v^{\prime}\right\rangle+Q_{0 w}(t) v(0)+Q_{1 w}(t) v(1)+\langle £(t), v\rangle=0, \\
w(x, 0)=u_{0}(x), \frac{\partial w}{\partial t}(x, 0)=u_{1}(x) \\
Q_{0 w}(t)=g_{0}(t)+\int_{0}^{t} k_{0}(t-s) w(0, s) d s \\
Q_{1 w}(t)=g_{1}(t)+\int_{0}^{t} k_{1}(t-s) w(1, s) d s
\end{array}\right.
$$

for each $v \in H^{1}(0,1)$ and a.e. time $0 \leq t \leq T$.

Define $\uplus: H^{1}\left(0, T ; L^{2}(0,1)\right) \rightarrow H^{1}\left(0, T ; L^{2}(0,1)\right)$ by setting

$$
\uplus u=w .
$$

It is claimed that if $T>0$ is small enough then $\uplus$ is a strict contraction. To prove this, let $u, \widetilde{u} \in H^{1}\left(0, T ; L^{2}(0,1)\right)$ arbitrarily and define $w=\uplus u, \widetilde{w}=\uplus \widetilde{u}$ as above. 
As a result, $w$ verifies $(3.7)$ for $£$ given by (3.2) and $\widetilde{w}$ satisfies the problem $(3.9)$

$$
\left\{\begin{array}{l}
\frac{d}{d t}\left\langle\frac{\partial \widetilde{w}}{\partial t}(t), v\right\rangle+\left\langle\mu(t) \frac{\partial \widetilde{w}}{\partial x}(t), v^{\prime}\right\rangle+\widetilde{Q_{0 w}}(t) v(0)+\widetilde{Q_{1 w}}(t) v(1)+\langle\widetilde{£}(t), v\rangle=0, \\
\widetilde{w}(x, 0)=u_{0}(x), \frac{\partial \widetilde{w}}{\partial t}(x, 0)=u_{1}(x), \\
\widetilde{Q_{0 w}}(t)=g_{0}(t)+\int_{0}^{t} k_{0}(t-s) \widetilde{w}(0, s) d s, \\
\widetilde{Q_{1 w}}(t)=g_{1}(t)+\int_{0}^{t} k_{1}(t-s) \widetilde{w}(1, s) d s, \\
\widetilde{£}(x, t):=G(\widetilde{u}(x, t))+H\left(\frac{\partial \widetilde{u}}{\partial t}(x, t)\right)-F(x, t),
\end{array}\right.
$$

for each $v \in H^{1}(0,1)$ and a.e. time $0 \leq t \leq T$. In addition, we have from (3.7) and (3.9), that

$$
\begin{aligned}
& \left\langle\frac{\partial^{2} w}{\partial t^{2}}(t)-\frac{\partial^{2} \widetilde{w}}{\partial t^{2}}(t), v\right\rangle+\left\langle\mu(t)\left[\frac{\partial w}{\partial x}(t)-\frac{\partial \widetilde{w}}{\partial x}(t)\right], v^{\prime}\right\rangle \\
& +\left(Q_{0 w}(t)-\widetilde{Q_{0 w}}(t)\right) v(0)+\left(Q_{1 w}(t)-\widetilde{Q_{1 w}}(t)\right) v(1)+\langle £(t)-\widetilde{£}(t), v\rangle=0,
\end{aligned}
$$

for each $v \in H^{1}(0,1)$ and a.e. time $0 \leq t \leq T$. Now, in (3.10), replacing $v$ by $\frac{\partial w}{\partial t}-\frac{\partial \widetilde{w}}{\partial t}$ and then integrating with respect to $t$, we get

$$
\begin{aligned}
\text { C }(t) & =\int_{0}^{t}\left\langle\frac{\partial \mu}{\partial s}(s),\left[\frac{\partial w}{\partial x}(s)-\frac{\partial \widetilde{w}}{\partial x}(s)\right]^{2}\right\rangle d s \\
& -2 \int_{0}^{t}\left[\frac{\partial w}{\partial s}(0, s)-\frac{\partial \widetilde{w}}{\partial s}(0, s)\right]\left(\int_{0}^{s} k_{0}(s-\tau)[w(0, \tau)-\widetilde{w}(0, \tau)] d \tau\right) d s \\
& -2 \int_{0}^{t}\left[\frac{\partial w}{\partial s}(1, s)-\frac{\partial \widetilde{w}}{\partial s}(1, s)\right]\left(\int_{0}^{s} k_{1}(s-\tau)[w(1, \tau)-\widetilde{w}(1, \tau)] d \tau\right) d s \\
& -2 \int_{0}^{t}\left\langle £(s)-\widetilde{£}(s), \frac{\partial w}{\partial s}(s)-\frac{\partial \widetilde{w}}{\partial s}(s)\right\rangle d s,
\end{aligned}
$$

in which

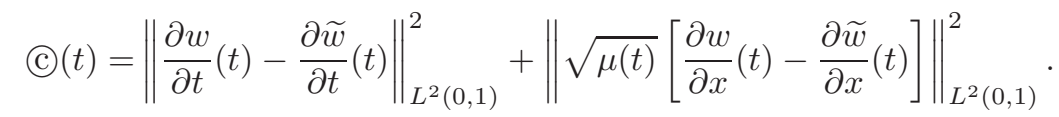


From $(2.1),(3.3),(3.11),(3.12)$ and assumptions $\left(A_{\mu}\right),\left(A_{k}\right)$, we deduce some following estimates:

$$
\begin{aligned}
& \int_{0}^{t}\left\langle\frac{\partial \mu}{\partial s}(s),\left[\frac{\partial w}{\partial x}(s)-\frac{\partial \widetilde{w}}{\partial x}(s)\right]^{2}\right\rangle d s \\
& \leq \frac{1}{\mu_{0}} \int_{0}^{t}\left\|\frac{\partial \mu}{\partial s}(s)\right\|_{L^{\infty}(0,1)} \text { C }(s) d s, \\
& -2 \int_{0}^{t}\left[\frac{\partial w}{\partial s}(i, s)-\frac{\partial \widetilde{w}}{\partial s}(i, s)\right]\left(\int_{0}^{s} k_{i}(s-\tau)[w(i, \tau)-\widetilde{w}(i, \tau)] d \tau\right) d s \\
& \leq \frac{2 \eta}{\mu_{0}} \mathrm{C}(t)+C_{i}\left(T, \mu_{0}, k_{i}\right) \int_{0}^{t} \mathrm{C}(s) d s, \\
& 2 \int_{0}^{t}\left\langle £(s)-\widetilde{£}(s), \frac{\partial w}{\partial s}(s)-\frac{\partial \widetilde{w}}{\partial s}(s)\right\rangle d s \\
& \leq \int_{0}^{t}\|£(s)-\widetilde{£}(s)\|_{L^{2}(0,1)}^{2} d s+\int_{0}^{t} \mathrm{C}(s) d s,
\end{aligned}
$$

for $i=0,1, \eta>0$, where

$$
\begin{aligned}
C_{i} & \left(T, \mu_{0}, k_{i}\right) \\
& =2 \eta T+\left(T^{2}+\frac{1}{\mu_{0}}\right)\left(2+2 \sqrt{2}\left|k_{i}(0)\right|+\frac{2}{\eta}\left\|k_{i}\right\|_{L^{2}(0, T)}^{2}+\left\|k_{i}^{\prime}\right\|_{L^{1}(0, T)}^{2}\right)
\end{aligned}
$$

are positive constants depending on $T, \mu_{0}$ and $k_{i}, i=0,1$. With the relevant choice of $\eta$, namely $\eta=\frac{\mu_{0}}{8}$, using Gronwall's inequality, we conclude from (3.11)-(3.16), that

$$
\text { C }(t) \leq\left(2 \int_{0}^{t}\|£(s)-\widetilde{£}(s)\|_{L^{2}(0,1)}^{2} d s\right) \exp [\AA(t)] \text {, }
$$

where

$$
\mathbb{R}(t)=2 \int_{0}^{t}\left(1+\sum_{i=0,1} C_{i}\left(T, \mu_{0}, k_{i}\right)+\frac{1}{\mu_{0}}\left\|\frac{\partial \mu}{\partial s}(s)\right\|_{L^{\infty}(0,1)}\right) d s .
$$

From $(3.16)$ and $\left(A_{\mu}\right)$, we deduce from (3.18) that there exists a constant $\mathbb{B}_{0}>0$ independent of $t$ such that

$$
2 \exp [\mathbb{R}(t)] \leq \mathbb{B}_{0} \text { for all } t \in[0, T] .
$$

From (3.19) and $\left(A_{G H}^{(2)}\right)$, we discover that (3.17) is equivalent to

$$
\text { (C) }(t) \leq 2 \AA_{0} \max \left\{K_{G}^{2}, K_{H}^{2}\right\}\|u-\widetilde{u}\|_{H^{1}\left(0, T ; L^{2}(0,1)\right)}^{2},
$$

for all $t \in[0, T]$ and $u, \widetilde{u} \in H^{1}\left(0, T ; L^{2}(0,1)\right)$ arbitrarily.

Combining (3.12) and (3.20), it follows that

$$
\left\|\frac{\partial w}{\partial t}(t)-\frac{\widetilde{w}}{\partial t}(t)\right\|_{L^{2}(0,1)}^{2} \leq 2 \Re_{0} \max \left\{K_{G}^{2}, K_{H}^{2}\right\}\|u-\widetilde{u}\|_{H^{1}\left(0, T ; L^{2}(0,1)\right)}^{2},
$$

for all $t \in[0, T]$ and $u, \widetilde{u} \in H^{1}\left(0, T ; L^{2}(0,1)\right)$ arbitrarily.

Moreover, it is not difficult to affirm that

$$
\|w(t)-\widetilde{w}(t)\|_{L^{2}(0,1)}^{2} \leq 2 \AA_{0} T^{2} \max \left\{K_{G}^{2}, K_{H}^{2}\right\}\|u-\widetilde{u}\|_{H^{1}\left(0, T ; L^{2}(0,1)\right)}^{2},
$$


for all $t \in[0, T]$ and $u, \widetilde{u} \in H^{1}\left(0, T ; L^{2}(0,1)\right)$ arbitrarily.

Hence, from (3.21) and (3.22), we deduce

$$
\|w-\widetilde{w}\|_{H^{1}\left(0, T ; L^{2}(0,1)\right)}^{2} \leq K_{H^{1}}^{2}\|u-\widetilde{u}\|_{H^{1}\left(0, T ; L^{2}(0,1)\right)}^{2}
$$

where $K_{H^{1}}=\sqrt{2 \mathbb{R}_{0}\left(T^{2}+1\right) \max \left\{K_{G}^{2}, K_{H}^{2}\right\}}$ and $u, \widetilde{u} \in H^{1}\left(0, T ; L^{2}(0,1)\right)$ arbitrarily. Thus,

$$
\|\uplus u-\uplus \widetilde{u}\|_{H^{1}\left(0, T ; L^{2}(0,1)\right)} \leq K_{H^{1}}\|u-\widetilde{u}\|_{H^{1}\left(0, T ; L^{2}(0,1)\right)},
$$

$u, \widetilde{u} \in H^{1}\left(0, T ; L^{2}(0,1)\right)$ arbitrarily. Therefore, $\uplus$ is a strict contraction, provided $T>0$ is so small that $K_{H^{1}}<1$.

As a result, with the application of Banach's fixed point theorem, we conclude that there exists $u \in H^{1}\left(0, T ; L^{2}(0,1)\right)$ which is the weak solution of problem (1.1)-(1.4).

In the case of $T>0$ given, we select $T_{1}>0$ so small that

$$
K_{H^{1}}=\sqrt{2 \AA_{0}\left(T_{1}^{2}+1\right) \max \left\{K_{G}^{2}, K_{H}^{2}\right\}}<1 .
$$

Then we are able to apply Banach's fixed point theorem to find a weak solution $u$ of problem (1.1)-(1.4) existing on the time interval $\left[0, T_{1}\right]$. Due to $u(\cdot, t), \frac{\partial u}{\partial t}(\cdot, t) \in$ $L^{2}(0,1)$ for a.e. $0 \leq t \leq T_{1}$, we can continue, upon redefining $T_{1}$ if necessary, by assuming $u\left(\cdot, T_{1}\right) \in H^{1}(0,1), \frac{\partial u}{\partial t}\left(\cdot, T_{1}\right) \in L^{2}(0,1)$. We can then repeat the above argument to extend our solution to the time interval $\left[T_{1}, 2 T_{1}\right]$. Continuing, after finite steps we construct a weak solution existing on the full interval $[0, T]$.

Step 2. The solvability in $L^{\infty}\left(0, T ; H^{1}(0,1)\right)$.

Step 1 shows that the operator $\uplus$ defined by

$$
\uplus u=w
$$

for all $u \in H^{1}\left(0, T ; L^{2}(0,1)\right)$ has at least one fixed point. Hence there exists $u \in H^{1}\left(0, T ; L^{2}(0,1)\right)$ such that $w=\uplus u \equiv u$. Then we can deduce from (3.6) that

$$
\left\|\frac{\partial u}{\partial t}(t)\right\|_{L^{2}(0,1)}^{2}+\left\|\sqrt{\mu(t)} \frac{\partial u}{\partial x}(t)\right\|_{L^{2}(0,1)}^{2} \leq C_{0} \text { for all } t \in[0, T] .
$$

In addition, from $(3.25)$ and $\left(A_{u}\right)$, it is easy to compute that

$$
\left\{\begin{array}{l}
\|u(t)\|_{L^{2}(0,1)}^{2} \leq 2\left\|u_{0}\right\|_{L^{2}(0,1)}^{2}+2 T^{2} \bigodot_{0} \leq \bigodot_{1}, \\
\left\|\frac{\partial u}{\partial x}(t)\right\|_{L^{2}(0,1)}^{2} \leq \frac{\bigodot_{0}}{\mu_{0}},
\end{array}\right.
$$

for all $t \in[0, T]$, where C $_{1}$ is also a positive constant independent of $t$.

After taking the essential supremum of the left sides of (3.26) with respect to $t$, we deduce that

$$
u \in L^{\infty}\left(0, T ; H^{1}(0,1)\right)
$$

As a result, problem (1.1)-(1.4) is also solvable on $L^{\infty}\left(0, T ; H^{1}(0,1)\right)$.

Step 3. The uniqueness of the weak solution.

To demonstrate uniqueness, suppose both $u$ and $\widetilde{u}$ are two weak solutions of problem (1.1)-(1.4) in $H^{1}\left(0, T ; L^{2}(0,1)\right) \cap L^{\infty}\left(0, T ; H^{1}(0,1)\right)$. Then we can have $w=u, \widetilde{w}=\widetilde{u}$ in (3.11), hence we discover

$$
\|u(t)-\widetilde{u}(t)\|_{H^{1}(0,1)}^{2} \leq \frac{2 \widetilde{\mathcal{C}_{0}}}{\min \left\{\frac{1}{T^{2}}, \mu_{0}\right\}} \int_{0}^{t}\|u(s)-\widetilde{u}(s)\|_{H^{1}(0,1)}^{2} d s,
$$


where

$$
\widetilde{\mathrm{C}_{0}} \geq 2 \exp \left(\int_{0}^{t}\left(3+\sum_{i=0,1} C_{i}\left(T, \mu_{0}, k_{i}\right)+\frac{1}{\mu_{0}}\left\|\frac{\partial \mu}{\partial s}(s)\right\|_{L^{\infty}(0,1)}\right) d s\right),
$$

Because of Gronwall's inequality, we deduce from (3.28), that

$$
\|u-\widetilde{u}\|_{L^{\infty}\left(0, T ; H^{1}(0,1)\right)}=0 .
$$

So, we obtain that $u \equiv \widetilde{u}$.

The above three steps show that the proof of the theorem is complete.

REMARK 2. When $H\left(\frac{\partial u}{\partial t}\right)=\lambda\left|\frac{\partial u}{\partial t}\right|^{p} \frac{\partial u}{\partial t}, p>0, \lambda<0$, it fails to apply FaedoGalerkin method for the unique solvability of problem (1.1)-(1.4). This method is only applicable for linear or some special nonlinear problems (as in [5], [10]-[20], $[24],[25])$.

REMARK 3. For some special nonlinear forms of damping-source terms $G(u)+$ $H\left(\frac{\partial u}{\partial t}\right)$ of problem (1.1)-(1.4) such as

$$
\begin{gathered}
K|u|^{p} u+\lambda\left|\frac{\partial u}{\partial t}\right|^{q} \frac{\partial u}{\partial t},(p, q) \in \mathbb{R}_{+}^{2} \backslash(0,0),(K, \lambda) \in \mathbb{R}_{+}^{2}, \\
G(u)+K \frac{\partial u}{\partial t}, K \in \mathbb{R},
\end{gathered}
$$

it is possible to apply Faedo-Galerkin approximation for the unique solvability of problem (1.1)-(1.4); however, some more assumptions for $\mu, G$ and $H$ must be added and unfortunately the proofs in such cases are usually not only very long but also truly complicated such as a priori estimates or passing to the limit by monotone techniques as in $[\mathbf{9}]$ and $[\mathbf{1 5}]$.

\section{The regularity of the weak solution}

In this section, the investigation for problem (1.1)-(1.4) is to study its weak solution's smoothness with respect to the smoothness of initial data. Specifically, assumptions $\left(A_{\mu}\right),\left(A_{G H}^{(1)}\right),\left(A_{G H}^{(2)}\right),\left(A_{F}\right),\left(A_{g}\right),\left(A_{k}\right)$, and $\left(A_{u}\right)$ are strengthened as follows:

$\left(\widetilde{A}_{\mu}\right) \mu \in C^{1}([0,1] \times[0, T]), \frac{\partial^{2} \mu}{\partial t^{2}} \in L^{2}\left(0, T ; L^{\infty}(0,1)\right), \mu(x, t) \geq \mu_{0}>0 ;$

$\left(\widetilde{A}_{G H}\right) G, H \in C^{1}(\mathbb{R}),\left|H^{\prime}(\eta)\right| \leq C_{H}|\eta|^{\alpha}, \forall \eta \in \mathbb{R}$, and $\alpha>0, C_{H} \geq 0$;

$\left(\widetilde{A}_{F}\right) F, \frac{\partial F}{\partial t} \in L^{2}((0,1) \times(0, T))$;

$\left(\widetilde{A}_{g}\right) g_{0}, g_{1} \in H^{2}(0, T)$;

$\left(\widetilde{A}_{k}\right) k_{0}, k_{1} \in W^{2,1}(0, T)$;

$\left(\widetilde{A}_{u}\right) u_{0} \in H^{2}(0,1), u_{1} \in H^{1}(0,1)$.

It is clear that problem (1.1)-(1.4) has a unique weak solution

$$
u \in H^{1}\left(0, T ; L^{2}(0,1)\right) \cap L^{\infty}\left(0, T ; H^{1}(0,1)\right)
$$

for which $\left(\widetilde{A}_{\mu}\right),\left(\widetilde{A}_{G H}\right),\left(\widetilde{A}_{F}\right),\left(\widetilde{A}_{g}\right),\left(\widetilde{A}_{k}\right)$, and $\left(\widetilde{A}_{u}\right)$ hold. The regularity of this weak solution is stated in the following theorem:

TheOrem 2. Let $\left(\widetilde{A}_{\mu}\right),\left(\widetilde{A}_{G H}\right),\left(\widetilde{A}_{F}\right),\left(\widetilde{A}_{g}\right),\left(\widetilde{A}_{k}\right)$, and $\left(\widetilde{A}_{u}\right)$ be hold. Then, for $T>0$, the problem (1.1)-(1.4) has a unique weak solution $u(x, t)$ satisfying

$$
u \in H^{2}\left(0, T ; L^{2}(0,1)\right) \cap H^{1}\left(0, T ; H^{1}(0,1)\right) \cap L^{\infty}\left(0, T ; H^{2}(0,1)\right) .
$$


Proof. From the previous section, $\left(A_{\mu}\right),\left(A_{G H}^{(1)}\right),\left(A_{G H}^{(2)}\right),\left(A_{F}\right),\left(A_{g}\right),\left(A_{k}\right)$, and $\left(A_{u}\right)$ ensure that problem (1.1)-(1.4) has a unique weak solution $u$ satisfying (3.1) and verifying the following variational problem

$$
\left\{\begin{array}{c}
\left\langle\frac{\partial^{2} u}{\partial t^{2}}(t), v\right\rangle+\left\langle\mu(t) \frac{\partial u}{\partial x}(t), v^{\prime}\right\rangle+Q_{0}(t) v(0)+Q_{1}(t) v(1) \\
\quad+\left\langle G(u(t))+H\left(\frac{\partial u}{\partial t}(t)\right), v\right\rangle=\langle F(t), v\rangle, \\
u(x, 0)=u_{0}(x), \frac{\partial u}{\partial t}(x, 0)=u_{1}(x) \\
Q_{0}(t)=g_{0}(t)+\int_{0}^{t} k_{0}(t-s) u(0, s) d s \\
Q_{1}(t)=g_{1}(t)+\int_{0}^{t} k_{1}(t-s) u(1, s) d s
\end{array}\right.
$$

for each $v \in H^{1}(0,1)$ and a.e. time $0 \leq t \leq T$. Certainly $\left(\widetilde{A}_{\mu}\right),\left(\widetilde{A}_{G H}\right),\left(\widetilde{A}_{F}\right),\left(\widetilde{A}_{g}\right)$, $\left(\widetilde{A}_{k}\right)$, and $\left(\widetilde{A}_{u}\right)$ also give the same result. Now, the matter is to prove that $u$ also satisfies (4.1). Indeed, differentiating $(4.2)_{1}$ with respect to $t$, replacing $v$ by $\frac{\partial^{2} u}{\partial t^{2}}$ and then integrating with respect to the time variable from 0 to $t$, we obtain that

$$
\begin{aligned}
(S(t)= & \left(S(0)+\int_{0}^{t}\left\langle\frac{\partial \mu}{\partial s}(s),\left|\frac{\partial^{2} u}{\partial x \partial s}(s)\right|^{2}\right\rangle d s\right. \\
& -2\left\langle\frac{\partial \mu}{\partial t}(t) \frac{\partial u}{\partial x}(t), \frac{\partial^{2} u}{\partial x \partial t}(t)\right\rangle+2\left\langle\frac{\partial \mu}{\partial t}(0) \frac{\partial u}{\partial x}(0), \frac{\partial^{2} u}{\partial x \partial t}(0)\right\rangle \\
& +2 \int_{0}^{t}\left\langle\frac{\partial^{2} \mu}{\partial s^{2}}(s) \frac{\partial u}{\partial x}(s), \frac{\partial^{2} u}{\partial x \partial s}(s)\right\rangle d s \\
& -2 \int_{0}^{t} Q_{0}^{\prime}(s) \frac{\partial^{2} u}{\partial s^{2}}(0, s) d s-2 \int_{0}^{t} Q_{1}^{\prime}(s) \frac{\partial^{2} u}{\partial s^{2}}(1, s) d s \\
& -2 \int_{0}^{t}\left\langle G^{\prime}(u(s)) \frac{\partial u}{\partial s}(s)+H^{\prime}\left(\frac{\partial u}{\partial s}(s)\right) \frac{\partial^{2} u}{\partial s^{2}}(s), \frac{\partial^{2} u}{\partial s^{2}}(s)\right\rangle d s \\
& +2 \int_{0}^{t}\left\langle\frac{\partial F}{\partial s}(s), \frac{\partial^{2} u}{\partial s^{2}}(s)\right\rangle d s,
\end{aligned}
$$

in which

$$
\text { (S) }(t)=\left\|\frac{\partial^{2} u}{\partial t^{2}}(t)\right\|_{L^{2}(0,1)}^{2}+\left\|\sqrt{\mu(t)} \frac{\partial^{2} u}{\partial x \partial t}(t)\right\|_{L^{2}(0,1)}^{2} .
$$

From $(2.1),(3.25),(4.3),(4.4)$ and assumptions $\left(\widetilde{A}_{\mu}\right),\left(\widetilde{A}_{G H}\right),\left(\widetilde{A}_{F}\right),\left(\widetilde{A}_{g}\right),\left(\widetilde{A}_{k}\right)$, and $\left(\widetilde{A}_{u}\right)$, we deduce some following estimates:

$$
\begin{gathered}
\text { S }(0)=\left\|\frac{\partial^{2} u}{\partial t^{2}}(0)\right\|_{L^{2}(0,1)}^{2}+\left\|\sqrt{\mu(0)} \frac{\partial^{2} u}{\partial x \partial t}(0)\right\|_{L^{2}(0,1)}^{2} \\
\leq 16\left(\left\|\frac{\partial \mu}{\partial x}(0)\right\|_{L^{\infty}(0,1)}\left\|u_{1}^{\prime}\right\|_{L^{2}(0,1)}^{2}+\mu_{0}\left\|u_{0}^{\prime \prime}\right\|_{L^{2}(0,1)}^{2}+\|G\|_{L^{2}(0,1)}^{2}\right. \\
\left.+\|H\|_{L^{2}(0,1)}^{2}+\|F(0)\|_{L^{2}(0,1)}^{2}\right)+\mu_{0}\left\|u_{1}^{\prime}\right\|_{L^{2}(0,1)}^{2}
\end{gathered}
$$




$$
\begin{aligned}
\int_{0}^{t}\left\langle\frac{\partial \mu}{\partial s}(s),\right| \frac{\partial^{2} u}{\partial x \partial s} & \left.\left.(s)\right|^{2}\right\rangle d s \\
& \leq \frac{1}{4 \mu_{0}^{2}} \int_{0}^{t}\left\|\frac{\partial \mu}{\partial s}(s)\right\|_{L^{\infty}(0,1)}^{2} d s+\int_{0}^{t}\left(S^{2}(s) d s\right.
\end{aligned}
$$

$$
\begin{aligned}
& -2\left\langle\frac{\partial \mu}{\partial t}(t) \frac{\partial u}{\partial x}(t), \frac{\partial^{2} u}{\partial x \partial t}(t)\right\rangle \leq\left\|\frac{\partial \mu}{\partial t}\right\|_{L^{\infty}((0,1) \times(0, T))}\left(\frac{\bigodot_{0}}{\mu_{0} \gamma}+\gamma(\mathrm{S}(t)),\right. \\
& 2\left\langle\frac{\partial \mu}{\partial t}(0) \frac{\partial u}{\partial x}(0), \frac{\partial^{2} u}{\partial x \partial t}(0)\right\rangle \leq 2\left\|\frac{\partial \mu}{\partial t}(0)\right\|_{L^{\infty}(0,1)}\left\|u_{0}\right\|_{L^{2}(0,1)}\left\|u_{1}^{\prime}\right\|_{L^{2}(0,1)},
\end{aligned}
$$

$2 \int_{0}^{t}\left\langle\frac{\partial^{2} \mu}{\partial s^{2}}(s) \frac{\partial u}{\partial x}(s), \frac{\partial^{2} u}{\partial x \partial s}(s)\right\rangle d s$

$$
\leq \frac{\mathrm{C}_{0}}{\mu_{0} \gamma}\left\|\frac{\partial^{2} \mu}{\partial t^{2}}\right\|_{L^{1}\left(0, T ; L^{\infty}(0,1)\right)}+\frac{\gamma^{2}}{4 \mu_{0}^{2}} \int_{0}^{t}\left\|\frac{\partial^{2} \mu}{\partial s^{2}}(s)\right\|_{L^{\infty}(0,1)}^{2} d s+\int_{0}^{t}\left(^{2}(s) d s,\right.
$$

$$
\begin{aligned}
& -2 \int_{0}^{t} Q_{i}^{\prime}(s) \frac{\partial^{2} u}{\partial s^{2}}(i, s) d s \leq D_{i}+\frac{2 \gamma}{\mu_{0}}\left(\mathrm{~S}(t)+\frac{1}{\mu_{0}} \int_{0}^{t}(S(s) d s,\right. \\
& -2 \int_{0}^{t}\left\langle G^{\prime}(u(s)) \frac{\partial u}{\partial s}(s), \frac{\partial^{2} u}{\partial s^{2}}(s)\right\rangle d s \\
& \leq T \subset C_{0}\left(\sup _{|\varepsilon| \leq\|u\|_{L^{\infty}\left(0, T ; H^{1}(0,1)\right)}}\left|G^{\prime}(\varepsilon)\right|\right)^{2}+\int_{0}^{t}(S(s) d s, \\
& -2 \int_{0}^{t}\left\langle H^{\prime}\left(\frac{\partial u}{\partial s}(s)\right) \frac{\partial^{2} u}{\partial s^{2}}(s), \frac{\partial^{2} u}{\partial s^{2}}(s)\right\rangle d s \\
& \leq 2 C_{H} \int_{0}^{t}\left(\bigodot_{0}+\frac{1}{\mu_{0}}(\mathrm{~S}(s))^{\frac{\alpha}{2}}(\mathrm{~S}(s) d s,\right. \\
& 2 \int_{0}^{t}\left\langle\frac{\partial F}{\partial s}(s), \frac{\partial^{2} u}{\partial s^{2}}(s)\right\rangle d s \leq\left\|\frac{\partial F}{\partial t}\right\|_{L^{2}((0,1) \times(0, T))}^{2}+\int_{0}^{t}(S(s) d s,
\end{aligned}
$$

in which $\gamma>0$ and $D_{i}$ are non-negative constants given by

$$
\begin{aligned}
D_{i}= & (2 \gamma+T) \bigodot_{0}+2\left|u_{1}(0)\right|\left(\left|g_{i}^{\prime}(0)\right|+\left|k_{i}(0) \| u_{0}(0)\right|\right) \\
& +\frac{1}{\gamma}\left[\left\|g_{i}^{\prime}\right\|_{C([0, T])}+\sqrt{2}\|u\|_{L^{\infty}\left(0, T ; H^{1}(0,1)\right)}\left(\left\|k_{i}^{\prime}\right\|_{L^{1}(0, T)}+\left|k_{i}(0)\right|\right)\right]^{2} \\
& +\int_{0}^{t}\left[\sqrt{2}\|u\|_{L^{\infty}\left(0, T ; H^{1}(0,1)\right)}\left(\left|k_{i}(0)\right|+\left|k_{i}^{\prime}(0)\right|+\left\|k_{i}^{\prime \prime}\right\|_{L^{1}(0, T)}\right)\right]^{2} d s
\end{aligned}
$$

for $i=0,1$.

In the sequel of (4.3)-(4.14) and by choosing $\gamma$ such that

$$
\gamma \leq \frac{1}{2\left\|\frac{\partial \mu}{\partial t}\right\|_{L^{\infty}((0,1) \times(0, T))}+\frac{4}{\mu_{0}}},
$$


we deduce that

$$
\mathrm{S}(t) \leq \mathrm{S}_{0}+\int_{0}^{t} K(\mathrm{~S}(s)) d s
$$

where

(4.16)

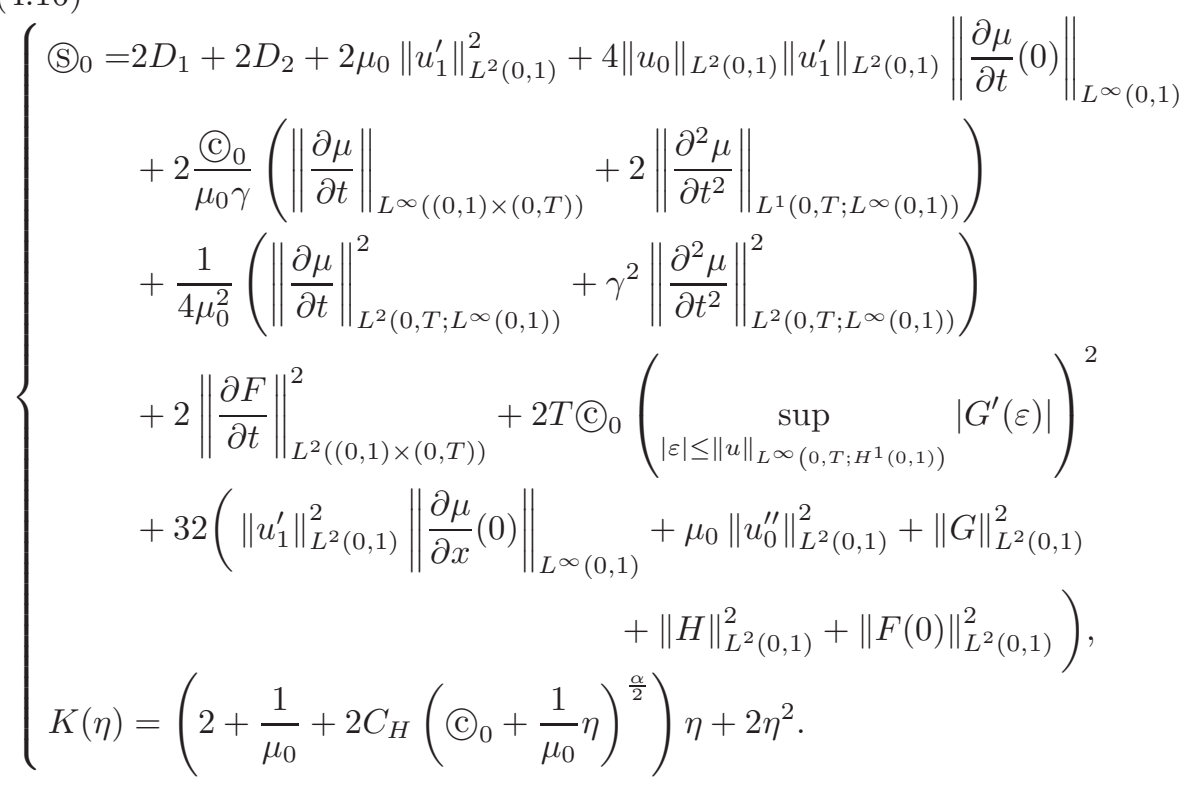

It is clear that $\mathrm{S}_{0}$ is a non-negative constant and $K(\eta)$ is non-decreasing for all $\eta \in[0, T]$. Thus, we deduce from (4.15) and (4.16), that

$$
\text { S }(t) \leq \mathrm{S}_{1}(t) \text { for all } t \in[0, T],
$$

where $S_{1}(t)$ is the maximal continuous solution of the nonlinear Volterra integral equation with non-decreasing kernel (see $[\mathbf{2}],[\mathbf{3}],[\mathbf{7}],[\mathbf{1 2}]$ ) on an interval $[0, T]$ given by

$$
\mathrm{S}_{1}(t)=\mathrm{S}_{0}+\int_{0}^{t} K\left(\mathrm{~S}_{1}(s)\right) d s .
$$

From (3.1), (4.4) and (4.17), we deduce that

$$
u \in H^{2}\left(0, T ; L^{2}(0,1)\right) \cap H^{1}\left(0, T ; H^{1}(0,1)\right) .
$$

In addition, we have from $(4.2),(4.18),\left(\widetilde{A}_{\mu}\right),\left(\widetilde{A}_{G H}\right)$, and $\left(\widetilde{A}_{F}\right)$, that

$$
\frac{\partial^{2} u}{\partial x^{2}}=\frac{1}{\mu}\left(\frac{\partial^{2} u}{\partial t^{2}}-\frac{\partial \mu}{\partial x} \frac{\partial u}{\partial x}+G(u)+H\left(\frac{\partial u}{\partial t}\right)-F\right) \in L^{\infty}\left(0, T ; L^{2}(0,1)\right)
$$

Hence, (3.1) and (4.19) verify that

$$
u \in L^{\infty}\left(0, T ; H^{2}(0,1)\right)
$$

Finally, it is clear that (4.18) and (4.20) make the expected proof complete.

REMARK 4. Without assumption $\left(\widetilde{A}_{G H}\right)$, we also obtain the regularity of the weak solution $u(x, t)$ of problem $(1.1)$-(1.4) with respect the assumptions $\left(\widetilde{A}_{\mu}\right)$, $\left(A_{G H}^{(1)}\right),\left(A_{G H}^{(2)}\right),\left(\widetilde{A}_{F}\right),\left(\widetilde{A}_{g}\right),\left(\widetilde{A}_{k}\right)$ and $\left(\widetilde{A}_{u}\right)$. However the smoothness of $u(x, t)$ in 
this case is less than what in Theorem 2. Specifically, we have the following theorem whose proof is similar to Theorem 1's and is obviously omitted:

TheOrEm 3. Let $\left(\widetilde{A}_{\mu}\right),\left(A_{G H}\right),\left(\widetilde{A}_{F}\right),\left(\widetilde{A}_{g}\right),\left(\widetilde{A}_{k}\right)$, and $\left(\widetilde{A}_{u}\right)$ hold. Then, for $T>0$, problem (1.1)-(1.4) has a unique weak solution $u(x, t)$ satisfying

$$
u \in C^{1}\left(0, T ; L^{2}(0,1)\right) \cap C\left(0, T ; H^{1}(0,1)\right) .
$$

From Lemma 2 and Lemma 3, we have that (4.21) can be deduced from (4.1), this means the regularity of the weak solution $u(x, t)$ by Theorem 2 is better that what in Theorem 3.

Remark 5. In the case $\frac{\partial^{2} \mu}{\partial t^{2}} \in L^{1}\left(0, T ; L^{\infty}(0,1)\right)$, Theorem 2 still holds but provided that $H^{\prime} \in L^{\infty}(\mathbb{R})$.

\section{References}

[1] Aassila, M. and Benaissa, A.: Existence of global solutions to a quasi-linear wave equation with general nonlinear damping, Electron. J. Differential Equations (91)(2002), pp. 22.

[2] Dang, A.D. and Alain, P.N.D.: Mixed problem for some semi-linear wave equation with a non-homogeneous condition, Nonlinear Anal. 12(6) (1998) 581-592.

[3] Burton, T.A.: Volterra Integral and Differential Equations, 2d ed., Elsevier B.V., Amsterdam, 2005.

[4] Benaddi, A. and Rao, B.: Energy decay rate of wave equations with indefinite damping, $J$. Differential Equations 161 (2000) 337-357.

[5] Bergounioux, M.; Nguyen, L.T. and Alain, P.N.D.: Mathematical model for a shock problem involving a linear viscoelastic bar, Nonlinear Anal. (13) (2001) 546-561.

[6] Brezis, H.: Analyse fonctionnelle: Théorie et applications, Masson, Paris, 1983.

[7] Collins, P.J.: Differential and Integral Equations, Oxford University Press, 2006.

[8] Evans, L.C.: Partial differential equations, Grad. Stud. Math., Amer. Math. Soc., 19, 1998.

[9] Lions, J.L.: Quelques méthodes de résolution des problèmes aux limites non-linéaires, DunodGauthier- Villars, Paris, 1969.

[10] Nguyen, L.T. and Alain, P.N.D.: A semilinear wave equation associated with a linear differential equation with Cauchy data, Nonlinear Anal. 24 (1995) 1261-1279.

[11] Nguyen, L.T.; Alain, P.N.D. and Tran, D.N.: On a shock problem involving a nonlinear viscoelastic bar, Bound. Value Probl. 3 (2005) 337-358.

[12] Lakshmikantham V. and Leela S.: Differential and Integral Inequalities, Academic Press, New York Vol. 1, 1969.

[13] Lê, Út V.: A contracted procedure for the unique solvability of a semilinear wave equation associated with a linear integral equation at the boundary, JP J. Fixed Point Theory and Appl. 3(1) (2008) 49-61.

[14] Lê, Út V.: The well-posedness of a semilinear wave equation associated with a linear integral equation at the boundary, Mem. Differential Equations Math. Phys. 44 (2008) 69-88.

[15] Lions, J.L. and Strauss, W.A.: Some nonlinear evolution equations, Bull. Soc. Math. France 93 (1965) 43-96.

[16] Nguyen, L.T.; Lê, Út V. and Nguyen, T.T.T.: A shock of a rigid body and a linear viscoelastic bar, Hcm Ped. Univ. J. Natur. Sci. 4(38) (2004) 27-40.

[17] Nguyen, L.T.; Lê, Út V. and Nguyen, T.T.T.: A shock of a rigid body and a linear viscoelastic bar: Global existence and stability of the solutions, Hcm Ped. Univ. J. Natur. Sci. 6(40) (2005) 26-39.

[18] Nguyen, L.T.; Lê, Út V. and Nguyen, T.T.T.: A shock problem involving a linear viscoelastic bar, Nonlinear Anal. 63 (2005) 198-224.

[19] Phung, K.D.: Polynomial decay rate for the dissipative wave equation, J. Differential Equations 240 (2007) 92-124.

[20] Santos, M.L.: Asymptotic expansion of solutions to wave equations with a memory condition at the boundary, Electron. J. Differential Equations, 73 (2001), 11 pp.

[21] Sengul, M.T.: An effective method for the existence of the global attractor of a nonlinear wave equation, Appl. Math. E-Notes 7 (2007) 179-185. 
[22] Takači, D. and Takači, A: On the approximate solution of a mathematical model of a viscoelastic bar, Nonlinear Anal. 67 (2007) 1560-1569.

[23] Teschl, G.: Nonlinear functional analysis, Lecture Notes in Math., Vienna Univ., Austria, 2001.

[24] Lê, Út V.; Truc, T.T.T. and Nguyen, L.T.: The shock of a rigid body and a linear viscoelastic bar: Global existence and stability of solutions, Proc. the $9^{\text {th }}$ Conf. Sci. Technol. Hcm Univ. Technol. 2005 24-29.

[25] Lê, Út V.; Truc, T.T.T. and Nguyen, L.T.: A shock of a rigid body and a linear viscoelastic bar: Global existence and Stability of the solutions, Vietnam J. Math. Appl. (2008) (accepted).

[26] Zeidler, E.: Nonlinear functional analysis and its applications, Vol. II, Springer-Verlag, Leipzig, 1989.

[27] Zheng, S: Nonlinear Evolution Equations, Chapman and Hall/CRC, 2004.

Department of Mathematical Sciences, P.O. Box 3000, FI-90014 University of Oulu, FINLAND

E-mail address: ut.van.le@oulu.fi

E-mail address: levanut@gmail.com

E-mail address: utlev@yahoo.com 